\title{
Seascape genomics of coastal bottlenose dolphins along strong gradients of temperature and salinity
}

\author{
Eleanor Pratt ${ }^{1}$, Luciano Beheregaray ${ }^{1}$, Kerstin Bilgmann ${ }^{2}$, Nikki Zanardo ${ }^{1}$, Fernando \\ Diaz-Aguirre $^{1}$, and Luciana Moller ${ }^{1}$ \\ ${ }^{1}$ Flinders University \\ ${ }^{2}$ Macquarie University
}

July 28, 2021

\begin{abstract}
Heterogeneous seascapes and strong environmental gradients in coastal waters are expected to influence adaptive divergence, particularly in species with large population sizes where selection is expected to be highly efficient. However, these influences might also extend to species characterized by strong social structure, natal philopatry and small home ranges. We implemented a seascape genomic study to test this hypothesis in Indo-Pacific bottlenose dolphins (Tursiops aduncus) distributed along the environmentally heterogeneous coast of southern Australia. The datasets included oceanographic and environmental variables thought to be good predictors of local adaptation in dolphins and 8,081 filtered single nucleotide polymorphisms (SNPs) genotyped for individuals sampled from six different bioregions. From a neutral perspective, population structure and connectivity of the dolphins were generally influenced by habitat type and social structuring. Genotype-environment association analysis identified 241 candidate adaptive loci and revealed that sea surface temperature and salinity gradients influenced adaptive divergence in these animals at both large- $(1,000 \mathrm{~s} \mathrm{~km})$ and fine-scales $(<100 \mathrm{~km})$. Enrichment analysis and annotation of candidate genes revealed functions related to sodium-activated ion transport, kidney development, adipogenesis and thermogenesis. The findings of spatial adaptive divergence and inferences of putative physiological adaptations challenge previous suggestions that marine megafauna is most likely to be affected by environmental and climatic changes via indirect, trophic effects. Our work contributes to conservation management of coastal bottlenose dolphins subjected to anthropogenic disturbance and to efforts of clarifying how seascape heterogeneity influences adaptive diversity and evolution in small cetaceans.
\end{abstract}

\section{Hosted file}

Seascape genomics of bottlenose dolphins.docx available at https://authorea.com/users/427954/ articles/532007-seascape-genomics-of-coastal-bottlenose-dolphins-along-strong-gradientsof-temperature-and-salinity 